\title{
Terahertz field control of interlayer transport modes in cuprate superconductors
}

\author{
Frank Schlawin, ${ }^{1, *}$ Anastasia S. D. Dietrich, ${ }^{1}$ Martin Kiffner, ${ }^{1,2}$ Andrea Cavalleri, ${ }^{1,3}$ and Dieter Jaksch ${ }^{1,2}$ \\ ${ }^{1}$ Clarendon Laboratory, University of Oxford, Parks Road, Oxford OX1 3PU, United Kingdom \\ ${ }^{2}$ Centre for Quantum Technologies, National University of Singapore, 117543 Singapore \\ ${ }^{3}$ Max Planck Institute for the Structure and Dynamics of Matter, Luruper Chaussee 149, 22761 Hamburg, Germany
}

(Received 3 May 2017; revised manuscript received 24 July 2017; published 28 August 2017)

\begin{abstract}
We theoretically show that terahertz pulses with controlled amplitude and frequency can be used to switch between stable transport modes in layered superconductors, modeled as stacks of Josephson junctions. We find pulse shapes that deterministically switch the transport mode between superconducting, resistive, and solitonic states. We develop a simple model that explains the switching mechanism as a destabilization of the center-of-mass excitation of the Josephson phase, made possible by the highly nonlinear nature of the light-matter coupling.
\end{abstract}

DOI: 10.1103/PhysRevB.96.064526

\section{INTRODUCTION}

Coherent control of the quantum dynamics in atomic or molecular systems forms an important pillar of modern quantum physics. Recent experimental progress in the generation and detection of terahertz radiation [1] expands this field from atomic ensembles to solid-state devices [2] and opens up unprecedented possibilities for the control and manipulation of macroscopic systems through light-matter interactions $[3,4]$. For instance, the nonlinear driving of phonon modes allows for the manipulation of electronic degrees of freedom in solids [5-9]. This coupling gives rise to a plethora of exciting effects, such as the melting of charge density waves [10-12], the excitation of synthetic magnetic fields [13], the possibility to drive metal-insulator transitions [14,15], control of heterointerfaces [16-18], or even the controlled creation of transient superconductivity [19-22].

A different type of nonlinearity arises in the $c$-axis electrodynamics of layered superconductors [23-32], which are well described by stacked, coupled Josephson junctions for temperatures sufficiently far below the critical temperature [33]. These systems exhibit a nonlinear coupling between external currents and Josephson plasmons, which is routinely employed for the creation of coherent terahertz radiation [34-44]. The inverse process, the light control of electric currents in layered superconductors, could offer exciting prospects for future quantum technologies. For example, optical driving could assist the flow of supercurrents in the presence of strong magnetic fields above $H_{c 1}$. Materials with high critical temperature $T_{c}$ like cuprates could then be used for applications where strong, superconducting currents need to be sustained to create high magnetic fields as, e.g., in magnetic resonance imaging [45]. However, this possibility of enhancing material properties by external driving has remained largely unexplored to date.

In this paper we consider a layered superconductor (sc) consisting of stacked two-dimensional sc layers as shown in Fig. 1(a). The material is driven by light polarized along the $z$ axis (which is parallel to the crystallographic $c$ axis), and the whole stack carries a dc current along the $z$ axis, which is smaller than the critical Josephson current $j_{J}$. We

\footnotetext{
*frank.schlawin@physics.ox.ac.uk
}

consider a parameter regime in which the system can occupy one of three states. The current can be transmitted either as a supercurrent, in which Cooper pairs tunnel between adjacent layers through the Josephson effect. It can also be transmitted as a quasiparticle current of individual charge carriers, with the corresponding voltage inducing plasma oscillations that can emit coherent light $[43,44]$. Additionally, solitonic solutions represent dynamical steady states in which quasiparticle and supercurrents coexist in the system. In contrast to [26,27], where stimulated emission due to external radiation was discussed, this paper explores the response to light in a regime where the pulse cannot be considered a perturbation of the undriven steady state.

We show how strong terahertz pulses can induce transitions between these three macroscopic quantum states. Since the plasmon dispersion depends on the macroscopic state, each state reacts differently to external driving, thus creating parameter regimes in which only one transition responds to the pulse, while other excitation paths remain "dark". Therefore, tailored pulses can act as deterministic switches between pairs of states. Roughly speaking, low-frequency driving destabilizes high-voltage states, while high-frequency radiation can force the system into high-voltage states. We explain this behavior by the light-induced destabilization of plasma oscillations in the center-of-mass mode.

The paper is organized as follows: Our model and the numerical approach are introduced in Sec. II. In Sec. III, we present simulations of the light-induced switching between macroscopic quantum states. These are put in a broader context in Sec. IV, where we explore the influence of pulse parameters and develop a simplified toy model to explain the destabilization mechanism of an initial state. Finally, we conclude with a discussion of the relevance of our results for future experiments in Sec. V.

\section{MODEL}

Our model for the setup shown in Fig. 1(a) follows from a description of the material in terms of the Josephson coupling between sc layers and macroscopic electromagnetism. The polarization of the pulses along the $z$ direction reduces the problem to dynamics along the $x$ and $z$ axis. The electromagnetic field couples to the gauge-invariant phase differences $\phi_{n}=\varphi_{n}-\varphi_{n+1}-\frac{2 \pi}{\Phi_{0}} \int_{n}^{n+1} d z A_{z}$ between adjacent 


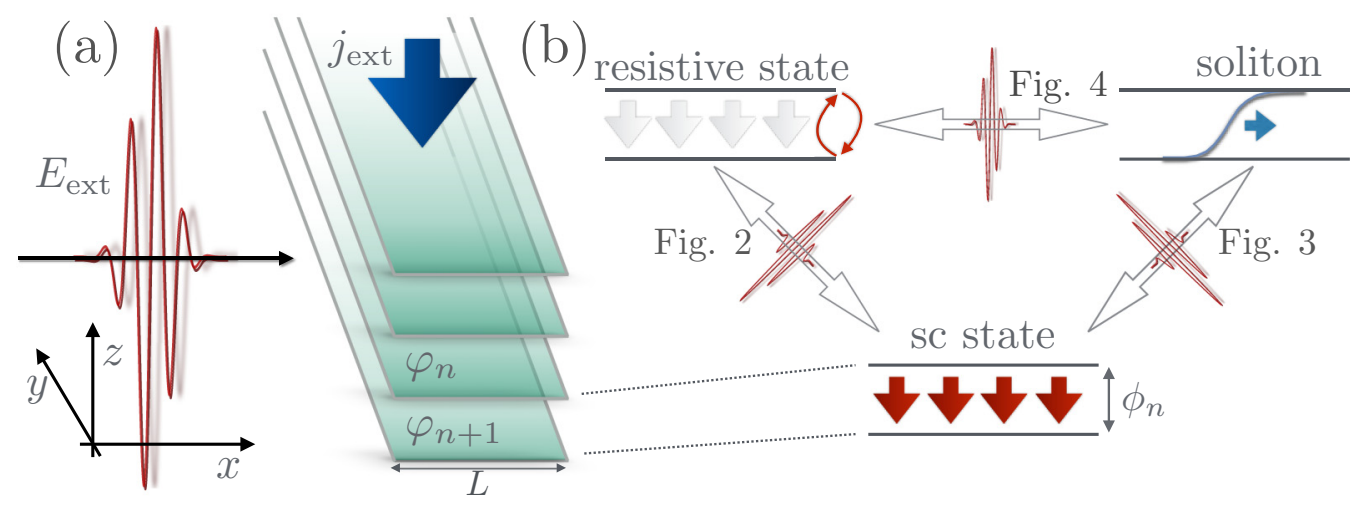

FIG. 1. (a) A short, layered superconductor is driven by a short laser pulse and a dc current $j_{\mathrm{ext}}$. (b) The interplay between the current, the nonlinearity of the crystal, and the light field $E_{\text {ext }}$ allows for the switching between the superconducting state, where the current flows as a supercurrent (red), the resistive state, where the current is supported by quasiparticles (gray), causing oscillatory supercurrents, and solitonic states, where quasiparticle and supercurrents coexist. Numerical results of simulations of these transitions are shown in Figs. 2, 3, and 4, respectively.

layers. Here, $A_{z}$ denotes the vector potential in $z$ direction, $\varphi_{n}$ the order parameter phase in the $n$th layer, and $\Phi_{0}$ the magnetic flux quantum. Its dynamics is coupled to the magnetic fields in the $y$ direction, since spatial changes of $\phi_{n}$ along the $x$ direction translate into a magnetic field perpendicular to it. Throughout this manuscript, we use dimensionless units, in which case the equations of motion may be written as $[46,47]$

$$
\begin{aligned}
& \frac{\partial^{2} \phi_{n}}{\partial \tau^{2}}+v_{c} \frac{\partial \phi_{n}}{\partial \tau}+\sin \phi_{n}-\frac{\partial h_{n}}{\partial \xi}+\eta(\xi, \tau)=j_{\text {ext }}, \\
& \left(\ell^{2} \nabla_{n}^{2}-1\right) h_{n}+\frac{\partial \phi_{n}}{\partial \xi}+v_{a b} \frac{\partial}{\partial \tau}\left(\frac{\partial \phi_{n}}{\partial \xi}-h_{n}\right)=0 .
\end{aligned}
$$

Here, $h_{n}$ denotes the dimensionless magnetic field, the damping constants $v_{c}$ and $v_{a b}$ are proportional to the quasiparticle conductivity along the $c$ axis and the $x y$ plane, respectively, and $\ell$ describes the strength of their magnetic coupling. Their expression in terms of physical quantities is given in Appendix A. The discrete $z$ derivative is defined as $\nabla_{n}^{2} h_{n} \equiv h_{n+1}+h_{n-1}-2 h_{n}$. The term $\sin \phi_{n}$ accounts for the Josephson coupling between the layers. In addition, we include the random driving term $\eta(\xi, \tau)$, emulating thermal phase fluctuations to ensure that our results are stable with respect to these fluctuations. In this paper, we focus on the low-temperature regime where these fluctuations are small. In particular, this means that they do not drive phase slips, nor do they excite thermal solitons. Any change in the macroscopic state is due to the external driving. Their impact on reflectance measurements is discussed in Appendix C. We approximate the boundary conditions for the layered structure $[36,48]$ by simple nonradiative conditions,

$$
\left.h_{n}(\tau)\right|_{\xi=0}=h_{\mathrm{ext}}(\tau)
$$

and

$$
\left.h_{n}(\tau)\right|_{\xi=L}=0,
$$

where $h_{\text {ext }}$ denotes the external pulse. These conditions represent excellent approximations, since the boundary electric field is suppressed by a large impedance mismatch to the vacuum [38]. We write the pulse as

$$
h_{\mathrm{ext}}(\tau)=A e^{-\left(\tau-\tau_{0}\right)^{2} /\left(2 \sigma^{2}\right)} \sin \left(\omega_{d r} \tau+\gamma\right),
$$

where $\omega_{d r}$ denotes the driving frequency, $\sigma$ the pulse duration, $\tau_{0}$ the pulse delay, $\gamma$ its carrier envelope phase (CEP), and $A$ its amplitude. Our parametrization is chosen such that $A$ denotes the maximal phase difference $\phi$ created by the pulse at the boundary at a given time. In order to chose realistic field strengths, we estimate [32] for LSCCO, according to which a phase difference $\phi \approx 1$ corresponds to a pulse with peak field intensity of $20 \mathrm{kV} / \mathrm{cm}$, with $100 \mathrm{kV} / \mathrm{cm}$ being within ouperimental reach. Thus we remain in the parameter regime where the of validity of the mean-field model has been tested experimentally [25]. The effects of light-induced pair breaking as well as microscopic materials details should be negligible. Furthermore, for the short pulses employed, heating effects do not play a role.

We consider a system which is sufficiently large along the $z$ axis, such that we can neglect finite-size effects due to coupling to connecting electrodes. As pointed out in Ref. [36], this is the case when the number of junctions $N$ exceeds the magnetic coupling length, i.e., $N \gtrsim \ell$. The external driving can then synchronize the dynamics in the junction, i.e., $\phi_{n} \rightarrow \phi$ and $h_{n} \rightarrow h$. This approximation was shown to yield excellent results in the simulation of the optical response of LSCCO in Ref. [32] and will also be used throughout this work. To check this assumption further, we have run simulations with up to 20 junctions and found that interlayer coupling does not alter the switching from superconductive to the resistive state.

Within our model, the value of $\ell$ becomes irrelevant in this limit and is set to $\ell=0$ in Eq. (2). We numerically solve Eqs. (1) and (2) using the method of lines (discretizing the spatial dimension on a grid with 250 points) and solving the resulting coupled ordinary equations with the IDA package [49] in MATHEMATICA. Furthermore, we limit our studies to the case where the interlayer voltage drop of two solitons would exceed the voltage drop of the resistive state. In this limit, only the three states shown in Fig. 1(b) exist. Increasing the length $L$ or the external current $j_{\text {ext }}$ to go beyond this 

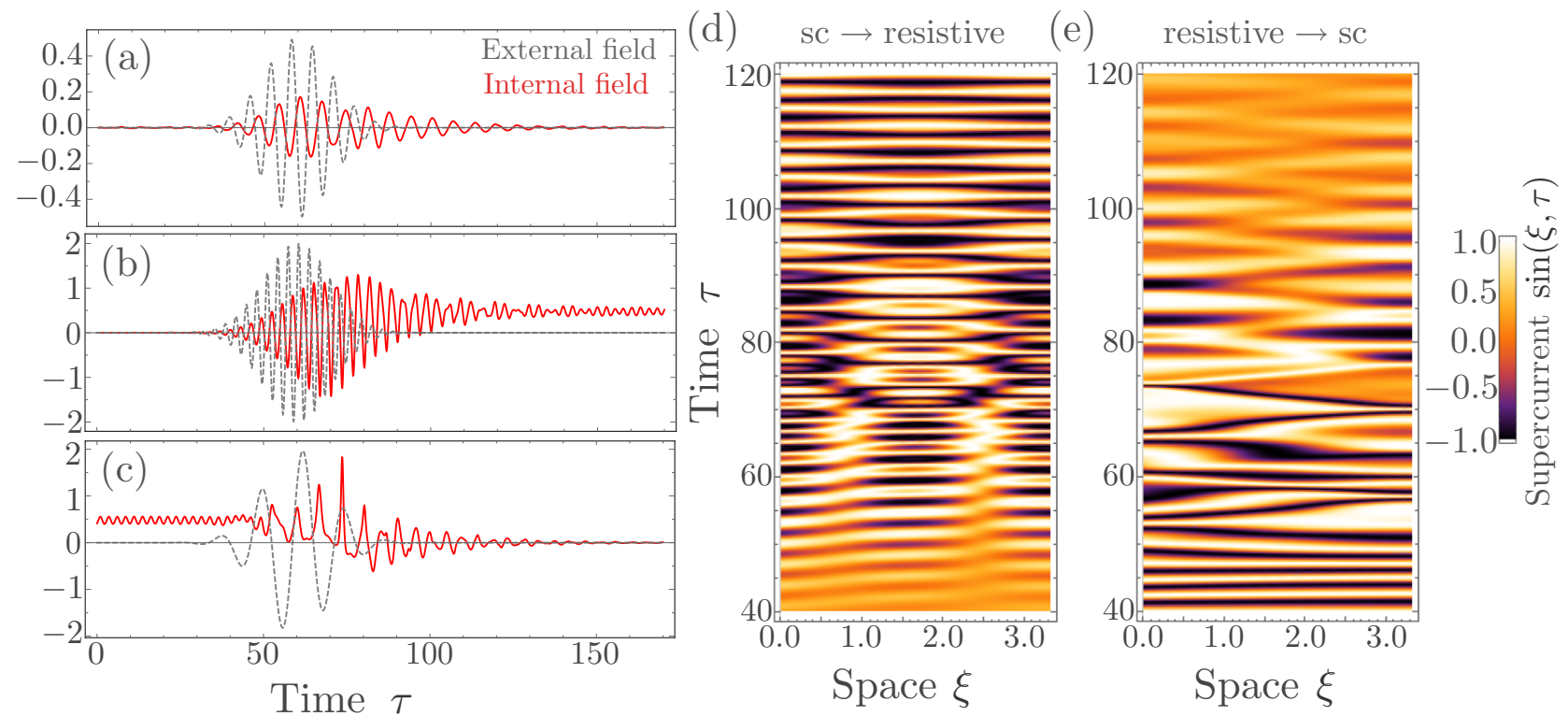

FIG. 2. Linear response and light-induced switching between sc and resistive state. (a) Electric field evolution (red) at the left boundary after excitation by a weak pulse (gray, dashed) at the plasma resonance, i.e., $\omega_{d r}=1$. The residual signal at large times stems from thermal fluctuations. (b) Excitation from an initial sc state by a strong pulse with $\omega_{d r}=2$ and amplitude $A=1.8$ that drives the system into the resistive state, signified by a constant electric field, i.e., the emergence of a voltage drop across the junctions. (c) Destabilization of the resistive state by another strong pulse (with $\omega_{d r}=0.5$ ), which disturbs the voltage drop and thus stops the quasiparticle current. (d) Supercurrent evolution $\sin (\phi(\xi, \tau))$ in the junction during the excitation process shown in panel (b). (e) Supercurrent evolution $\sin (\phi(\xi, \tau))$ during the destabilization process shown in panel (c). We fix the values $v_{c}=v_{a b}=0.1$ and $j_{\text {ext }}=0.25, L=3.3$, as well as the pulse parameters $\tau_{0}=60$ and $\gamma=0$ in the simulations.

limit would allow states with several solitons (but not add qualitatively new physics within our model).

\section{LIGHT-INDUCED DYNAMICS}

In this section we numerically study the light-induced transitions between sc, resistive, and solitonic states shown in Fig. 1(b). We first discuss the linear response to weak pulses when the system is initialized in the sc state, given by $\phi_{\mathrm{sc}}=\arcsin j_{\text {ext }}$ and $h=0$. The optical signature of the coherent Josephson coupling in this state consists of a sharp edge in the reflectivity spectrum. Weak pulses near this plasma edge excite plasma oscillations as shown in Fig. 2(a), where we depict the external field $h_{\text {ext }}(\tau)$ (gray) and the internal electric field $d \phi / d \tau$. The field oscillation follows the driving pulse with phase difference $\pi$, indicating the absorption of energy from the external field. The Fourier transform of the reflected field with respect to $\tau$ shows that the plasma resonance peak is located at $\left(1-j_{\text {ext }}^{2}\right)^{1 / 2}$ [50]. We present simulations of reflectivity signals of such weak pulses in Appendix C. In Fig. 9, the plasma edge is seen as a sharp reduction of the reflected signal frequency component. Thus, the system absorbs energy very efficiently at this frequency. Far from the plasma edge, $\omega_{d r} \gg 1$ or $\omega_{d r} \ll 1$, weak waves cannot penetrate the system [28]. As we shall see in the following, this changes dramatically for stronger pulses when the linear response no longer applies. The system cannot be treated as an effective medium that is not affected by the light, and strong plasma oscillations can actively influence the state of the superconductor.

\section{A. Switching between superconducting and resistive transport}

We first focus on the transition between the sc and the resistive state. Figure 2(b) shows the interaction of a strong pulse with the system in the sc state. The strong pulse excites plasma oscillations which no longer disperse but instead build up in magnitude and stabilize uniform plasma oscillations. This can be seen in Fig. 2(d), where we depict the supercurrent evolution $\propto \sin \phi(\xi, \tau)$ across the entire junction, and where traveling waves can be distinguished at short times from the stable uniform oscillations after the interaction with the pulse. A finite-voltage drop is stabilized, the constant offset of the electric field in Fig. 2(b) when averaged over the weak oscillations, satisfying the Ohmic relation $d \phi / d \tau=j_{\text {ext }} / \nu_{c}$ [51]. The macroscopic state is now given approximately by the McCumber state [52],

$$
\phi_{\mathrm{res}}(\tau)=\omega_{0} \tau+\Im\left\{\frac{e^{i \omega_{0} \tau}}{\omega_{0}^{2}-i \omega_{0} \nu_{c}}\right\},
$$

where $\omega_{0}=j_{\text {ext }} / v_{c}$. The weak oscillations on top of the offset in Fig. 2(b) stem from Cooper pair tunneling induced by the ac Josephson relation and are described by the second term in Eq. (6). The pulse has thus switched the system from the sc to the resistive state.

Strong laser pulses may also be employed to destabilize the quasiparticle current by disturbing the voltage drop across the junction and thereby switching the system to the sc state. An example is given in Figs. 2(c) and 2(e), where a pulse drives the system initialized in the resistive state (6). In contrast to the sc state, the resistive state does not show a plasma edge (see Appendix B) and low-frequency waves $(\omega<1)$ can penetrate 


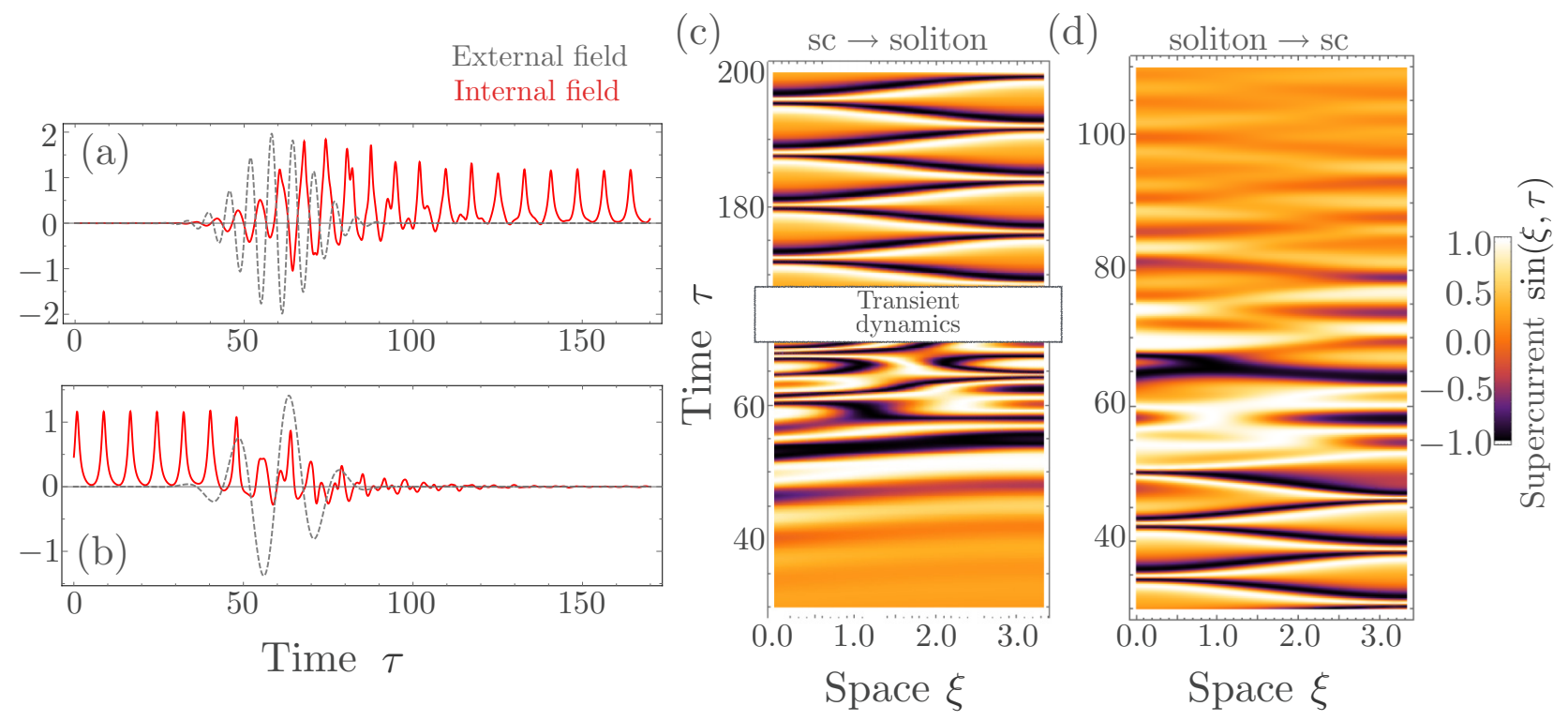

FIG. 3. Light-induced switching between sc and solitonic state. (a) Electric field evolution (red) at the left boundary during the excitation by a pulse with $A=2$ and $\omega_{d r}=1$ that drives the system from the sc into the solitonic state. (b) The inverse transition from the solitonic to the sc state is induced by a pulse with amplitude $A=1.5$ and frequency $\omega_{d r}=0.4$. (c) Supercurrent evolution during the optical excitation of a traveling soliton by the same pulse as in panel (a). (d) Supercurrent evolution during the destruction of the soliton by the same pulse as in panel (b). The remaining parameters are identical to Fig. 2.

the system. The driving frequency is too small to directly couple to the resistive state plasma oscillations with $\omega_{0}=$ $j_{\text {ext }} / v_{c} \simeq 2.5$. However, as can be seen in Fig. 2(c), when the electric field becomes negative, it locally cancels the voltage drop and thereby stops the quasiparticle current. This in turn destabilizes the oscillations and eventually destroys them. As one can see in panel 2(e), the first weaker oscillation of the incoming pulse at $\tau \simeq 50$ [compare with Fig. 2(b)] shifts the phase of the resistive state's uniform oscillations. The second, stronger oscillation at $\tau \simeq 60$ disturbs the voltage such that the fast plasma oscillations collapse and finally decay on a time scale $\sim v_{c}^{-1}$. Thus, this pulse has switched the system back into the sc state.

\section{B. Switching between superconducting and solitonic transport}

Figure 3 shows the driving of the sc state by a strong pulse which excites the solitonic state. Here, after a longer transient evolution which we skip in Fig. 3(c), the nonlinearity induces a traveling soliton. This soliton represents a different kind of stable dynamical state in which the currents are carried by both quasiparticle and supercurrent contributions. Moving Josephson solitons are quantum vortices of the condensate surrounded by supercurrents which carry one magnetic flux quantum with them. In a spatially infinite medium, in the absence of external currents and dissipation, their wave function is given by [52]

$$
\phi_{\text {soliton }}(\xi, \tau)=4 \tan ^{-1}\left[\exp \left( \pm \frac{\xi-u \tau}{\sqrt{1-u^{2}}}\right)\right],
$$

with the velocity $|u| \leqslant 1$. The solitonic wave, Eq. (7), describes a traveling steplike increase of the phase by $2 \pi$ and thereby drives the supercurrent $\sin \phi(\xi, \tau)$ through a full cycle, see Fig. 3(a). It can be shown that, in a first approximation, external current and dissipation do not significantly alter this shape but merely affect the velocity by supplying or draining kinetic energy, respectively [52]. At equilibrium, such an analysis yields the so-called power-balance velocities, $u_{\infty}=$ $\pm 1 /\left(1+\left[4 v_{c} /\left(\pi j_{\text {ext }}\right)\right]^{2}\right)^{1 / 2}$, which in turn allow us to compute the fundamental frequency of the kink motion, $\omega_{\infty} \simeq 2 \pi \frac{u_{\infty}}{L}$. Whenever a soliton hits the boundary, it is reflected as an antisoliton (which also increases the phase while moving in the opposite direction), emitting a burst of radiation [43] and thus providing a direct experimental fingerprint for its creation by the driving pulse. Averaged over one oscillation period, it amounts to a voltage drop of $V / \omega_{p}=\hbar \omega_{\infty} /(2 e)$, which is smaller than the voltage drop for the resistive state $\hbar \omega_{0} /(2 e)$.

The soliton may also be destroyed by optical means. This is exemplified in Fig. 3(b), where a pulse disturbs the soliton such that it disperses into plasma wave packets which quickly decay. Similar to the resistive state, we find the soliton to be unstable against driving below the plasma edge.

\section{Switching between solitonic and resistive states}

In Fig. 4, we show the switching between solitonic and resistive states. Figures 4(a) and 4(c) depict the interaction of a junction in the solitonic state with a strong pulse that switches the sc to the resistive state. The solitonic state is signified by bursts of radiation that are emitted whenever the soliton hits the junction boundary. As can be seen in Fig. 4(a), these bursts are stopped by the pulse and a constant voltage drop [with small oscillations on top, see Eq. (6)] is stabilized instead. In the supercurrent plot of panel 4(c), this change is reflected in the destruction of the traveling phase slip and the emergence of uniform plasma oscillations across the entire junction.

Conversely, a pulse with the same driving frequency that excited a soliton from the sc state can disrupt the voltage and 

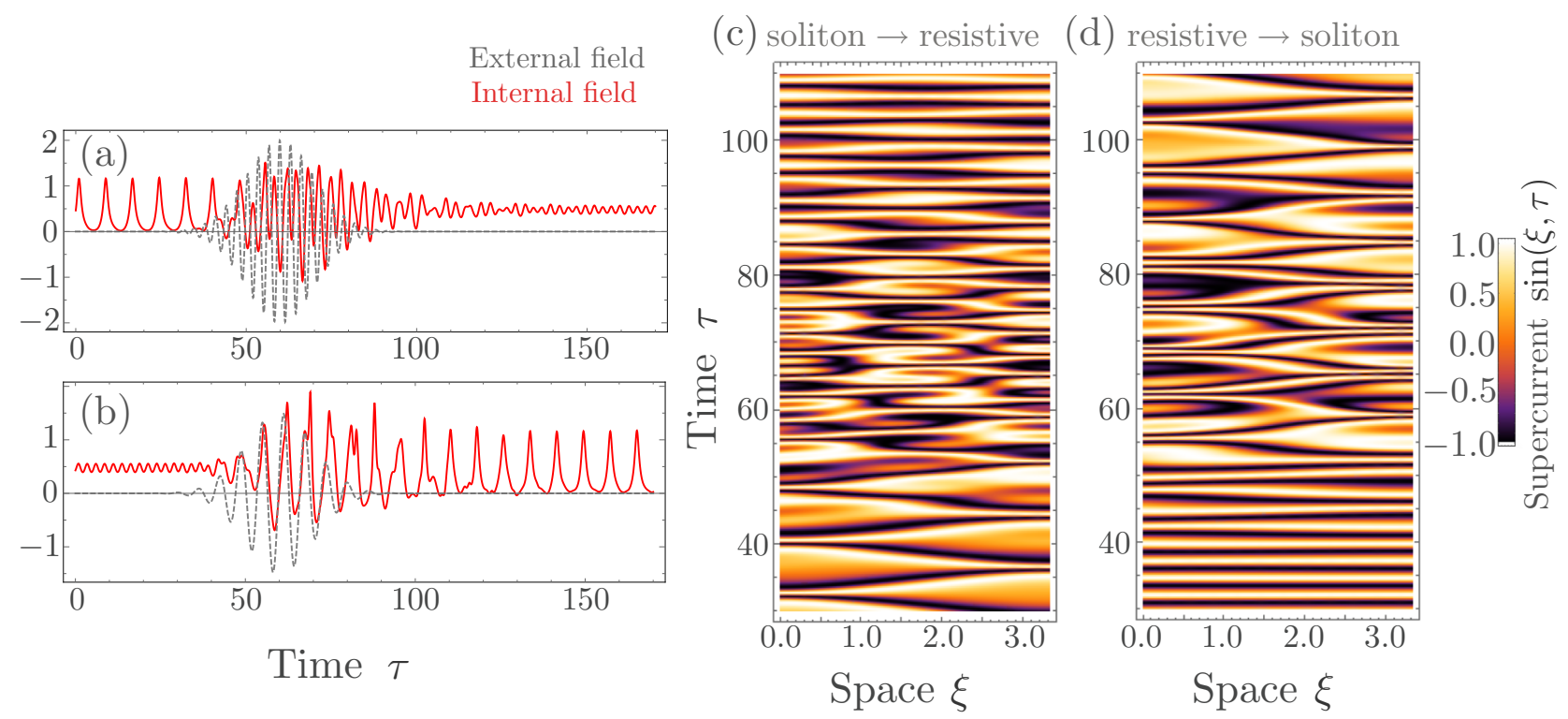

FIG. 4. (a) Electric field evolution (red) at the left boundary after excitation by a pulse with $A=2$ and $\omega_{d r}=2$ (i.e., with the same parameters as the pulse in Fig. 2 that switches the sc to the resistive state) that drives the system from the solitonic into the resistive state, signified by a constant electric field, i.e., the emergence of a voltage drop across the junctions. (b) The inverse transition from the resistive to the solitonic state is induced by a pulse with amplitude $A=1.5$ and frequency $\omega_{d r}=1$ (like a pulse that creates a soliton from the sc state). (c) Supercurrent evolution $\sin (\phi(\xi, \tau))$ in the junction during the excitation process shown in panel (a). (d) Supercurrent evolution sin $(\phi(\xi, \tau))$ during the destabilization shown in panel (b). The remaining parameters are identical to Fig. 2.

switch the system from the resistive into the solitonic state [see Figs. 4(b) and 4(d)].

\section{PARAMETER DEPENDENCE OF THE FINAL STATES AND SWITCHING MECHANISM}

We now investigate systematically the parameter space spanned by the driving frequency $\omega_{d r}$ and the amplitude $A$ in Eq. (5). This will allow us to explain the mechanism underlying the switching between different states. We first discuss the destabilization of the sc state, then of the resistive state, and finally of the soliton.

\section{A. Destabilization of the sc state}

Figure 5(a) shows the final state of the system after driving the sc state by a pulse with amplitude $A$ and frequency $\omega_{d r}$. We identify three resonances that destabilize the sc state at low driving strengths and define two distinct regions: For $\omega_{d r} \leqslant 1.5$, solitonic states are excited predominantly, with tiny islands of resistive and sc states in between. In contrast, in the region $\omega_{d r} \gtrsim 1.8$ the pulses excite solely resistive states (as exemplified in Fig. 2, which falls into this region of parameter space). While the boundary between the latter region and the sc region appears regular, the former region is fairly irregular, with closely intertwined sc and solitonic solutions. Despite these irregularities, Fig. 5 demonstrates that wide regions in parameter space exist in which solitonic or resistive states can be excited deterministically.

Next we establish a simple model for the explanation of the results shown in Fig. 5(a). We derive the analytic solution of the linearized equation of motion for the phase in Appendix B and find that it exhibits pronounced peaks at the eigenmodes $k_{n}=n \pi / L$ of the undriven system. Therefore, to gain a better understanding of the structure in Fig. 5(a), we make the ansatz

$$
\phi(\xi, \tau)=\phi_{\mathrm{sc}}+\sum_{n} f_{n}(\tau) \cos \left(\frac{n \pi \xi}{L}\right),
$$

where we recall the sc state $\phi_{\mathrm{sc}}=\arcsin \left(j_{\mathrm{ext}}\right)$ and expand the Josephson coupling to leading nonlinear order, $\sin \left(\phi_{\mathrm{sc}}+\delta\right) \simeq$ $\sin \left(\phi_{\mathrm{sc}}\right)+\cos \left(\phi_{\mathrm{sc}}\right) \delta-\sin \left(\phi_{\mathrm{sc}}\right) \delta^{2} / 2$. The linear order $\cos \left(\phi_{\mathrm{sc}}\right) \delta$ merely yields decoupled wave equations for the $k$ modes with the potential $\left[\left(1-j_{\mathrm{ext}}^{2}\right)^{1 / 2}+k_{n}^{2}\right] f_{n}^{2} / 2$. The next order,
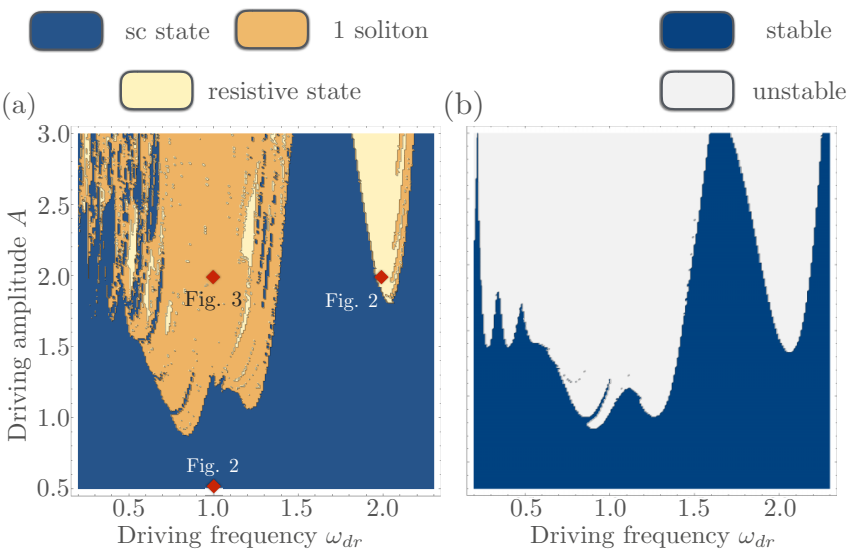

FIG. 5. (a) Final macroscopic quantum state after excitation by a pulse with inverse bandwidth $\sigma=10$, amplitude $A$, and carrier frequency $\omega_{d r}$. The system is initialized in the sc state. The parameters of Figs. 2 and 3 are indicated by red diamonds. Blue regions indicate parameters in which the system remains in the sc state, and the pulse merely induces transient plasma waves. Dark (bright) ochre regions indicate parameters in which traveling solitons (resistive states) are excited. (b) Destabilization of the COM mode according to Eq. (9). 
$\sin \left(\phi_{\mathrm{sc}}\right) \delta^{2} / 2$, has two effects: First, it couples the various eigenmodes' equations of motion such that the resonant excitation of a specific mode can influence the system at all frequencies. Second, it also changes the potential of the center-of-mass (COM) mode $n=0$ to a cubic one, $(1-$ $\left.j_{\text {ext }}^{2}\right)^{1 / 2} f_{0}^{2} / 2-j_{\text {ext }} f_{0}^{3} / 3$, which features a stable equilibrium point at $f_{0}^{*}=0$, as before, but further adds an unstable one at $f_{0}^{* *}=\left(1-j_{\text {ext }}^{2}\right)^{1 / 2} / j_{\text {ext }}$. When the excitation exceeds this point, the dynamics would become unbounded to leading nonlinear order. This light-induced destabilization of fluctuations around the steady state signifies the possible switching to a different macroscopic quantum state. The COM equation of motion then reads

$$
f_{0}^{\prime \prime}(\tau)+v_{c} f_{0}^{\prime}(\tau)+\sqrt{1-j_{\mathrm{ext}}^{2}} f_{0}(\tau)-\frac{j_{\mathrm{ext}}}{2} f_{0}^{2}(\tau)=f_{d r}(\tau),
$$

where $f_{d r}(\tau)$ describes the driving of the COM mode. As discussed above, the driving $f_{d r}$ consists of two contributionsthe direct excitation by the light pulse as well as the indirect excitation through the nonlinear coupling to other modes [see Eqs. (B15)-(B17) for details]. We model the external driving as identical to the external pulse, $A \sin \left(\omega_{d r} \tau\right) \exp \left[-\tau^{2} /\left(2 \sigma^{2}\right)\right]$. This does not capture details of the pulse propagation in the system and deviations must be expected, in particular at large driving amplitudes, where the full system is expected to saturate. Nevertheless, is sufficient to understand the main physical properties of the full model, as shown below.

As shown in Fig. 5(b), the simple model Eq. (9) is able to reproduce the different parameter regimes shown in panel (a) very well. While it cannot reproduce the irregular specklelike patterns below $\omega_{d r}<0.5$ of the full model, it does correctly reproduce the three resonances. The deviation at low frequencies originates from the simplified model allowing excitations at low frequencies while the full model predicts almost complete reflection, with excitations only being permitted above the nonlinear supratransmission threshold [53]. The simplified model further allows associating the resonances with the resonant excitation of the $k$ modes: The lowest-energy resonance stems predominantly from the direct excitation of the $n=0(\mathrm{COM})$ mode. The other two resonances originate from indirect excitation via the quadratic coupling to the $n=1$ mode and to the $n=2$ mode, respectively.

The good agreement between Figs. 5(a) and 5(b) demonstrates that the switching between macroscopic states may be understood as the light-induced destabilization of the COM mode. Our simple model gives rise to correct predictions not only for the excitation of the uniform resistive state, but also for the highly localized moving soliton state where intuition could suggest a close connection to high- $k$ modes. However, our explanation is insufficient to predict the final state following the destabilization.

\section{B. Destabilization of the resistive state}

The parameter space for the destabilization of the resistive state is shown in Fig. 6(a). It demonstrates that the resistive state can be destabilized by low-frequency irradiation with driving frequency $\omega_{d r} \lesssim 1.5$, while it remains stable against high-frequency pulses. We again compare these simulations
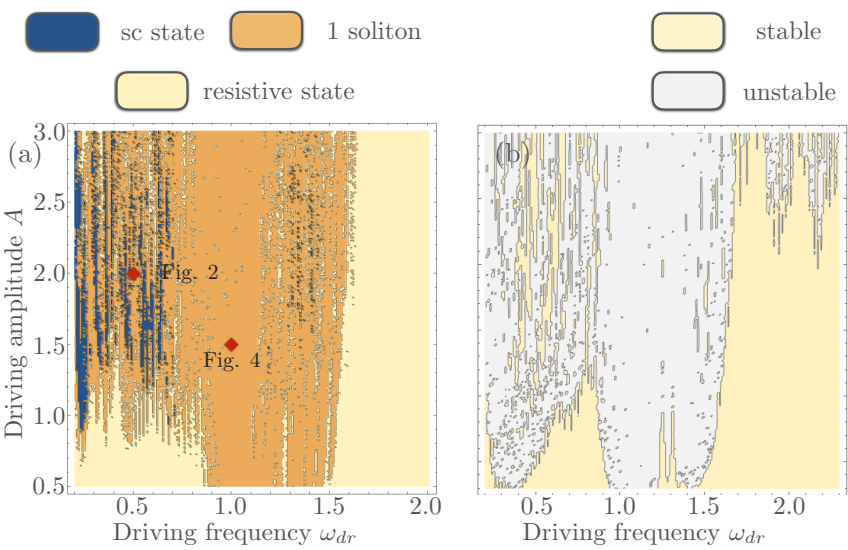

FIG. 6. (a) Final macroscopic quantum state after excitation by a pulse with inverse bandwidth $\sigma=10$, amplitude $A$, and carrier frequency $\omega_{d r}$. The system is initialized in the resistive state. (b) Destabilization of the COM mode according to Eqs. (B19)-(B21).

with results from a simplified model, which we derive in Eqs. (B19)-(B21), shown in panel (b). Just like in the case of the sc state, it overestimates the instability at very low frequencies, $\omega_{d r} \lesssim 0.5$, and further predicts instabilities above $\omega_{d r} \gtrsim 1.5$. The latter can be explained by an overestimation of the maximal Josephson current at large driving: Since we introduce the driving directly into the equations of motion, the excitation increases linearly with the amplitude $A$. Due to the nonlinear Josephson coupling, this is not the case in the full dynamics, and the simplified model thus overestimates the instability. Yet it correctly predicts the transition frequency $\omega_{d r} \approx$ 1.5 , as well as the comparatively large amplitude needed to destabilize the resistive state for $\omega_{d r} \lesssim 0.5$. Like for the sc state, it is the destabilization of the center-of-mass mode that is responsible for the switching between macroscopic states.

Although the destabilization mechanism is the same, the resulting final state structures in the parameter space are vastly different compared to the sc state in Fig. 5. This is due to the different dispersion relations, which are linear, $\omega=|k|$, in the resistive state, and in contrast feature a band gap, $\omega^{2}=$ $\sqrt{1-j_{\text {ext }}^{2}}+k^{2}$, in the sc state. Hence, the all-important centerof-mass mode shifts to zero frequency, rendering the resistive state susceptible to low-frequency driving and stable against high-frequency perturbations.

Furthermore, we remark that both the results from the simulations and those from the simplified model are highly sensitive on the driving frequency $\omega_{d r}$. A small change in the driving frequency can result in a different final state (or change the dynamics from stable to unstable in the simplified model), whereas small changes of the driving amplitude seldom change the dynamics. As we will discuss next in the context of the destabilization of the soliton, whose parameter space shows similar features, the use of single- or few-cycle pulses creates more regular structures, since their larger bandwidth effectively averages over a frequency interval, resulting in larger intervals with a unique final state.

\section{Destabilization of the soliton}

Figure 7 presents the final state after the interaction with a pulse with constant pulse duration $\sigma=10$ (i.e., as before). It 


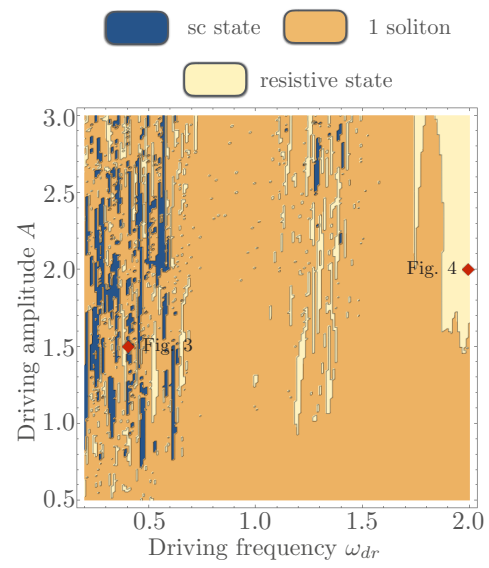

FIG. 7. Final macroscopic quantum state after excitation by a pulse with inverse bandwidth $\sigma=10$, amplitude $A$, and carrier frequency $\omega_{d r}$. The system is initialized in the soliton state.

demonstrates that it is in fact possible to destroy the soliton and reset the system to the sc state (blue regions) or the resistive state (bright yellow regions). The soliton is mostly vulnerable against low-frequency driving, with $\omega_{d r} \lesssim 0.6$, but also against very-high-frequency excitation, with $\omega_{d r} \gtrsim 1.8$. Yet, the structure appears irregular and shows the same vertical structure along the vertical axis we observed in Fig. 6, where small changes of $\omega_{d r}$ can change the final state. With the exception of driving at very high frequencies, one cannot identify large regions where the soliton can be destroyed with confidence.

As the soliton is a strongly localized wave, its interaction with short pulses differs from the previous two cases in that the CEP $\gamma$ in Eq. (5) may become important. Thus, if we instead simulate the interaction with single-cycle pulses of duration $\sigma=2 / \omega_{d r}$, we obtain the results shown in Fig. 8. In these plots, we also pick $\tau_{0}=10 / \omega_{d r}$ to assure that pulses with different driving frequencies have the same shape, which is shown on the right side of the panels. The oscillation frequency of the soliton $\omega_{\infty}$ is considerably larger than the driving frequency $\omega_{d r}$, such that the value of $\tau_{0}$ is not central to the results. We find that the larger bandwidth at lower frequencies averages out the irregular structure of Fig. 7. This creates regions in which the switching can be accomplished with confidence. In contrast to the earlier results in Fig. 7, the final state after excitation by these short pulses strongly depends on the phase. In our simulation, the phase $\gamma=0$ in panel (a) favors the excitation of the resistive state. While at intermediate values in panel (b) it can only seldom destroy the soliton, at larger values in panel (c) it favors the excitation of the sc state.

This behavior can be understood qualitatively through the analysis of the pulse form, which is shown for each case on the left of the panels. Note that for each set of parameters $\left\{\omega_{d r}, A\right\}$, this pulse is stretched or compressed in both time and amplitude, but it always retains this shape. At $\gamma=0$, negative values of the field amplitude dominate the pulse form. A negative magnetic field at the left boundary implies that $\partial h / \partial \xi>0$, as long as no other magnetic fields are present. In Eq. (1), this lowers the value of the left-hand side of the equation. Thus, the quasiparticle current term $v_{c} \partial \phi / \partial \tau$ has to rise such that the sum of the terms equals the external current. Conversely, at $\gamma=\pi$, positive values dominate in the pulse, thus reducing the instantaneous voltage and thereby favoring the excitation of the sc state.

\section{DISCUSSION AND CONCLUSIONS}

In conclusion, we have proposed to manipulate the macroscopic quantum state of a current-carrying layered
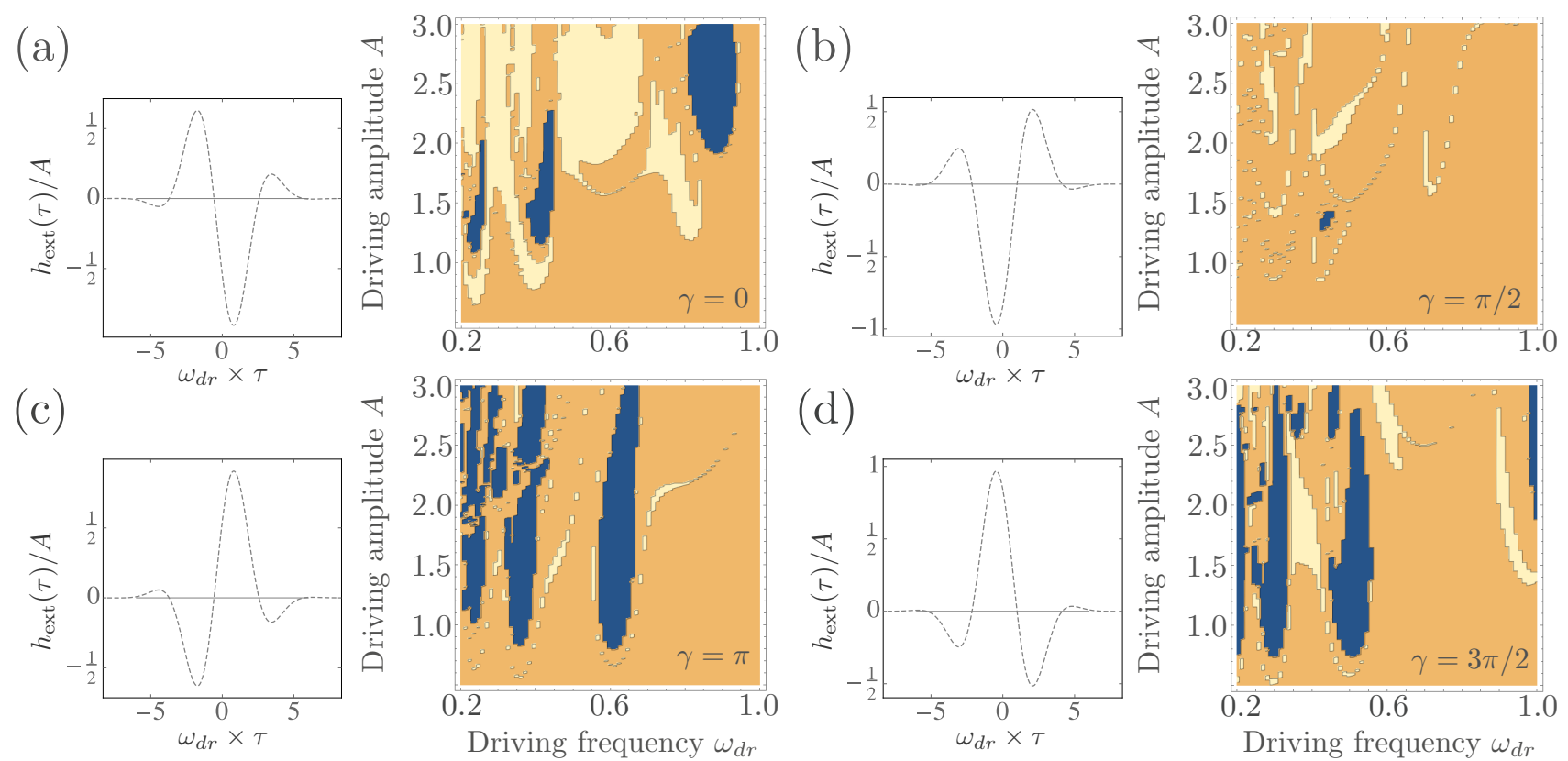

FIG. 8. Final macroscopic quantum state after excitation by a single cycle pulse with bandwidth $\sigma=2 / \omega_{d r}$ and CEP (a) $\gamma=0$, (b) $\pi / 2$, (c) $\pi$, and (d) $3 \pi / 2$. The system is initialized in the soliton state (same as Fig. 7). The panels on the left indicate the pulse form for the given $\mathrm{CEP}$, which is identical for any frequency. 
superconductor using terahertz pulses. By focusing on the interaction with strong, few-cycle pulses, we investigated their use as ultrafast switches that can reset the system from the zero-voltage sc state to a finite-voltage state (either solitonic or resistive) and vice versa. We showed that this manipulation is enabled by the strong nonlinearity of the light-matter interaction in the system by means of a simple toy model. Here, the nonlinearity results in a driving term for the center-of-mass mode of the plasma oscillations, whose destabilization indicates the switching between macroscopic states. We have pointed out possible applications of these findings.

Driving the system below the plasma resonance does not affect the sc state below the supratransmission threshold [53], but it can destabilize the resistive or solitonic state. For instance, both pulses shown in Figs. 2(c) and 3(b) do not destabilize the sc state, yet they can destabilize the resistive state and thereby prohibit phase fluctuations from destroying the coherence between junctions. Our work thus points towards an unusual, yet feasible approach to the ongoing effort to laser cool superconducting fluctuations [54-56]. Similarly, it will be interesting to explore other parameter regimes supporting different macroscopic states and investigate, for instance, whether driving can destroy or stabilize vortex lattices in the presence of external magnetic fields. This will be pursued in future work.

\section{ACKNOWLEDGMENTS}

D.J. thanks the Graduate School of Excellence Material Science in Mainz for hospitality during part of this work. The research leading to these results has received funding from the European Research Council under the European Union's Seventh Framework Programme (FP7/2007-2013) Grant Agreement No. 319286 Q-MAC. M.K. thanks the National Research Foundation and the Ministry of Education of Singapore for support.

\section{APPENDIX A: PHYSICAL UNITS}

The $c$-axis electrodynamics of layered superconductors is determined by the dielectric constant $\epsilon$ and three characteristic length scales: the penetration depths $\lambda_{c}$ along its $c$ axis, and $\lambda_{a b}$ along the $a b$ planes, as well as the interlayer spacing $s$. From these we can construct the Josephson plasma frequency

$$
\omega_{p}=\frac{c}{\sqrt{\epsilon} \lambda_{c}},
$$

the number of magnetically coupled junctions

$$
\ell=\frac{\lambda_{a b}}{s},
$$

and the anisotropy parameter $\gamma=\lambda_{a b} / \lambda_{c}$. All currents in this paper are normalized to the critical Josephson current, which is given by $j_{J}=c \Phi_{0} /\left(8 \pi^{2} s \lambda_{c}\right)$ [47]. Time is measured in units of the plasma frequency, i.e., $\tau=\omega_{p} \times t$, and the spatial coordinate in units of the $c$-axis penetration length $\xi=x / \lambda_{c}$. Furthermore, the quasiparticle conductivities along the $c$ axis $\sigma_{c}$ and the $a b$ plane $\sigma_{a b}$ are converted into dimensionless damping rates by the relations

$$
\begin{aligned}
v_{c} & =\frac{4 \pi \sigma_{c}}{\epsilon \omega_{p}}, \\
v_{a b} & =\frac{4 \pi \sigma_{a b}}{\epsilon \omega_{p} \gamma^{2}} .
\end{aligned}
$$

The dimensionless magnetic field $h_{n}$ is measured in units of $B_{0}=\Phi_{0} /\left(2 \pi \lambda_{c} s\right)$. The electric field is given by [47]

$$
\begin{aligned}
E_{z} & =\frac{\Phi_{0}}{2 \pi c s} \frac{\partial \phi_{n}}{\partial t} \\
& =\frac{B_{0}}{\sqrt{\epsilon}} \frac{\partial \phi_{n}}{\partial \tau} .
\end{aligned}
$$

Gaussian units are employed.

\section{APPENDIX B: CENTER-OF-MASS MODE DYNAMICS}

Here we derive the simplified equations of motion of eigenmode fluctuations around a given steady state of the system $\phi_{0}$. To this end, we write the full wave function as a sum,

$$
\phi(\xi, \tau)=\phi_{0}+\phi_{\epsilon}(\xi, \tau),
$$

where $\phi_{0}$ denotes a dynamical steady-state solution of the full mode, which could be either the sc state $\phi_{\mathrm{sc}}$, the resistive state (6), or the soliton (7), and $\left|\phi_{\epsilon}\right| \ll\left|\phi_{0}\right|$. Inserting this ansatz into the sine-Gordon equation, we obtain to second order

$$
\frac{\partial^{2} \phi_{\epsilon}}{\partial \tau^{2}}+v_{c} \frac{\partial \phi_{\epsilon}}{\partial \tau}+\cos \left(\phi_{0}\right) \phi_{\epsilon}-\frac{\partial^{2} \phi_{\epsilon}}{\partial \xi^{2}}=\frac{1}{2} \sin \left(\phi_{0}\right) \phi_{\epsilon}^{2} .
$$

The left-hand side of Eq. (B2) describes the propagation of linear waves with the system in the stated state $\phi_{0}$. The righthand side yields corrections to this wave behavior at larger amplitudes.

\section{Linear waves}

\section{a. sc state}

In the sc state, we have $\cos \left(\phi_{\mathrm{sc}}\right)=\left(1-j_{\text {ext }}^{2}\right)^{1 / 2}$ and $\sin \left(\phi_{\text {sc }}\right)=j_{\text {ext }}$. In this case, the left-hand side of Eq. (B2) represents a linear wave equation which we can solve straightforwardly by Fourier transform. We seek a solution of the linearized sine-Gordon equation,

$$
\frac{\partial^{2} \phi_{\epsilon}}{\partial \tau^{2}}+v_{c} \frac{\partial \phi_{\epsilon}}{\partial \tau}+\sqrt{1-j_{\text {ext }}^{2}} \phi_{\epsilon}-\frac{\partial^{2} \phi_{\epsilon}}{\partial \xi^{2}}=0,
$$

subject to the boundary conditions

$$
\begin{gathered}
\left.\frac{\partial \phi_{\epsilon}}{\partial \xi}\right|_{\xi=0}=h_{\text {ext }}(\tau), \\
\left.\frac{\partial \phi_{\epsilon}}{\partial \xi}\right|_{\xi=L}=0,
\end{gathered}
$$

with $h_{\text {ext }}$ given by Eq. (5). Writing the wave function as

$$
\phi_{\epsilon}(\xi, \tau)=\int d \omega \Im e^{i \omega \tau} \phi_{\omega}(\xi)
$$


with

$$
\phi_{\omega}(\xi)=A \cos k_{\omega} \xi+B \sin k_{\omega} \xi,
$$

we obtain from Eq. (B3) the dispersion relation

$$
\omega^{2}-i v_{c} \omega=\sqrt{1-j_{\mathrm{ext}}^{2}}+k_{\omega}^{2},
$$

and from the boundary conditions (B4) and (B5),

$$
\begin{gathered}
B=\frac{h_{\mathrm{ext}}(\omega)}{2 \pi k_{\omega}}, \\
A=B \tan ^{-1} k_{\omega} L .
\end{gathered}
$$

Thus, we arrive at

$$
\begin{aligned}
& \phi_{\epsilon}(\xi, \tau) \\
& \quad=\int d \omega \Im e^{i \omega \tau} \frac{h_{\mathrm{ext}}(\omega)}{2 \pi k_{\omega}}\left[\tan ^{-1}\left(k_{\omega} L\right) \cos \left(k_{\omega} \xi\right)+\sin \left(k_{\omega} \xi\right)\right] .
\end{aligned}
$$

Clearly, this solution is strongly peaked whenever $k_{\omega}=n \pi / L$, i.e., when the driving excites a cavity resonance in the junction.

\section{b. Resistive state}

We approximate the resistive state (6) by its dominant term $\phi_{\text {resistive }} \simeq \omega_{0} \tau$ to obtain the Mathieu equation

$$
\frac{\partial^{2} \phi_{\epsilon}}{\partial \tau^{2}}+v_{c} \frac{\partial \phi_{\epsilon}}{\partial \tau}+\cos \left(\omega_{o} \tau\right) \phi_{\epsilon}-\frac{\partial^{2} \phi_{\epsilon}}{\partial \xi^{2}}=0 .
$$

With the parameters employed in this manuscript, we have $\omega_{0}=2.5$; hence it is much larger than frequencies around and below the plasma resonance with $\omega \leqslant 1.0$ that we are most interested in. Therefore there will be very little mixing between these widely disparate frequencies, and in a first approximation, we replace the highly oscillatory term $\cos \left(\omega_{0} \tau\right)$ by its time-averaged value $=0$. This creates a free-space-like wave equation with linear dispersion:

$$
\omega^{2}-i v_{c} \omega=k_{\omega}^{2} .
$$

Whereas Eq. (B8) only supports (approximately) real wave vectors above the plasma edge, when $\omega>\left(1-j_{\mathrm{ext}}^{2}\right)^{1 / 2}$ there is no forbidden spectral region in the resistive state and lowfrequency waves can penetrate the system.

\section{c. Solitonic state}

In an infinitely long junction, a soliton breaks the timetransversal symmetry. This creates a Goldstone mode at zero frequency, while the remaining dispersion relation is not affected by the presence of the soliton [57]. Hence, the existence of the zero-frequency mode can explain the susceptibility of the soliton state against low-frequency driving.

\section{Destabilization of the sc state}

The linear wave dispersion (B8) motivates us to reduce our description to only the first few eigenmodes with $n=0,1,2$ in the investigation of the nonlinear corrections to the solution (B11). [This is also supported by the reflectivity spectrum in
Fig. 9(a).] We write

$$
\phi_{\epsilon}(\xi, \tau)=\sum_{n=0}^{2} f_{n}(\tau) \cos \left(\frac{n \pi \xi}{L}\right),
$$

multiply Eq. (B2) by $\cos (n \pi x / L)$, and integrate over space. This approach results in the coupled equations of motion of the eigenmodes:

$$
\begin{aligned}
& f_{0}^{\prime \prime}(\tau)+v_{c} f_{0}^{\prime}(\tau)+\sqrt{1-j_{\mathrm{ext}}^{2}} f_{0}(\tau)-\frac{j_{\mathrm{ext}}}{2} f_{0}^{2}(\tau) \\
& =\frac{j_{\mathrm{ext}}}{4}\left[f_{1}^{2}(\tau)+f_{2}^{2}(\tau)\right]+f_{0 d r}(\tau), \\
& f_{1}^{\prime \prime}(\tau)+v_{c} f_{1}^{\prime}(\tau)+\left[\sqrt{1-j_{\mathrm{ext}}^{2}}+\left(\frac{\pi}{L}\right)^{2}\right] f_{1}(\tau) \\
& =j_{\mathrm{ext}}\left[f_{0}(\tau) f_{1}(\tau)+\frac{1}{2} f_{1}(\tau) f_{2}(\tau)\right]+f_{1 d r}(\tau), \\
& f_{2}^{\prime \prime}(\tau)+v_{c} f_{2}^{\prime}(\tau)+\left[\sqrt{1-j_{\mathrm{ext}}^{2}}+\left(\frac{2 \pi}{L}\right)^{2}\right] f_{2}(\tau) \\
& =j_{\mathrm{ext}}\left[f_{0}(\tau) f_{2}(\tau)+\frac{1}{4} f_{1}^{2}(\tau)\right]+f_{2 d r}(\tau) .
\end{aligned}
$$

We have neglected the coupling to higher- $n$ modes and added phenomenological driving terms which we simply write as

$$
2 f_{0 d r}(\tau)=f_{1 d r}(\tau)=f_{2 d r}(\tau)=A \sin \left(\omega_{d r} \tau\right) e^{-\left(\tau-\tau_{0}\right)^{2} /\left(2 \sigma^{2}\right)},
$$

i.e., we assume that they have the same shape as the external driving (5). The additional factor 2 ahead of $f_{0 d r}$ stems from the spatial integration, since $\int d \xi 1=L$ and $\int d x \cos ^{2}\left(k_{n} x\right)=$ $L / 2$, thus giving greater weight to the finite- $k$ modes. We stress that this approach neglects the details of the wave-packet propagation inside the Josephson junctions, as these details are not essential for the understanding of the switching process.

\section{Destabilization of the resistive state}

As in Eq. (B12), we approximate the resistive state (6) by its dominant term $\phi_{\text {resistive }} \simeq \omega_{0} \tau$. Inserting the approximation into Eq. (B2), the same approach as above yields

$$
\begin{aligned}
& f_{0}^{\prime \prime}(\tau)+v_{c} f_{0}^{\prime}(\tau)+\cos \left(\phi_{\mathrm{res}}(\tau)\right) f_{0}(\tau)-\frac{\sin \left(\phi_{\mathrm{res}}(\tau)\right)}{2} f_{0}^{2}(\tau) \\
& =\frac{\sin \left(\phi_{\mathrm{res}}(\tau)\right)}{4}\left[f_{1}^{2}(\tau)+f_{2}^{2}(\tau)\right]+f_{0 d r}(\tau), \\
& f_{1}^{\prime \prime}(\tau)+v_{c} f_{1}^{\prime}(\tau)+\left[\cos \left(\phi_{\mathrm{res}}(\tau)\right)+\left(\frac{\pi}{L}\right)^{2}\right] f_{1}(\tau) \\
& =\sin \left(\phi_{\mathrm{res}}(\tau)\right)\left[f_{0}(\tau) f_{1}(\tau)+\frac{1}{2} f_{1}(\tau) f_{2}(\tau)\right]+f_{1 d r}(\tau),
\end{aligned}
$$

$$
\begin{array}{r}
f_{2}^{\prime \prime}(\tau)+v_{c} f_{2}^{\prime}(\tau)+\left[\cos \left(\phi_{\mathrm{res}}(\tau)\right)+\left(\frac{2 \pi}{L}\right)^{2}\right] f_{2}(\tau) \\
=\sin \left(\phi_{\mathrm{res}}(\tau)\right)\left[f_{0}(\tau) f_{2}(\tau)+\frac{1}{4} f_{1}^{2}(\tau)\right]+f_{2 d r}(\tau) .
\end{array}
$$



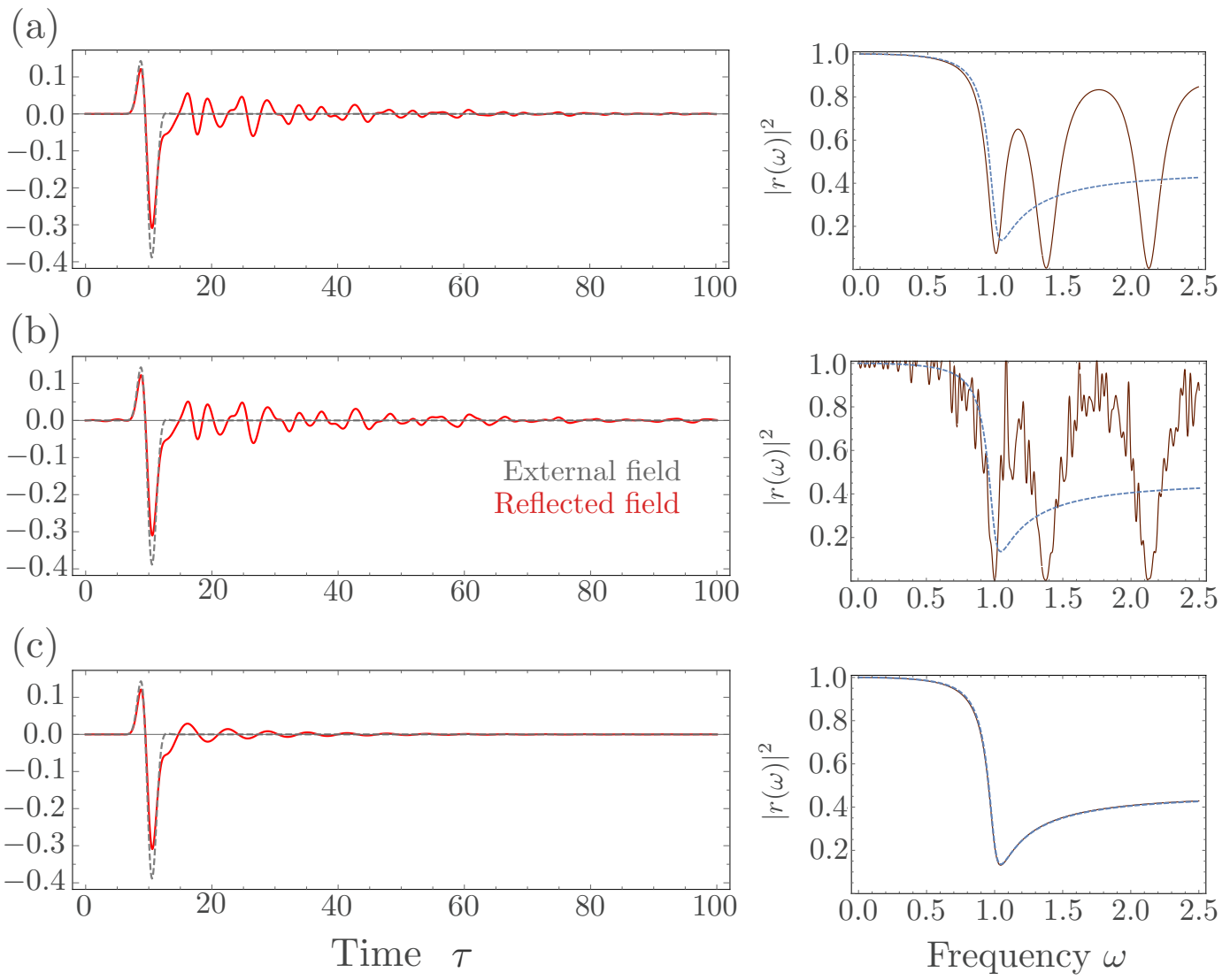

FIG. 9. Optical signals and noise. (a) Time evolution (left) and reflectance $|r(\omega)|^{2}$ (right) of a weak excitation in a short junction without noise. The blue, dashed line in the reflectance plot shows the theoretical expectation for the bulk system according to Eq. (C3). (b) The same including the noise level used in the paper. (c) Time evolution and reflectance in a long junction. The simulations and theoretical expectation coincide and cannot be distinguished.

We write the driving fields as

$4 f_{0 d r}(\tau)=2 f_{1 d r}(\tau)=2 f_{2 d r}(\tau)=A \sin \left(\omega_{d r} \tau\right) e^{-\left(\tau-\tau_{0}\right)^{2} /\left(2 \sigma^{2}\right)}$,

where the factor 2 is inserted to roughly match the simulations of the full model.

\section{APPENDIX C: FLUCTUATIONS}

In the absence of external currents, the only ground state is the sc state. We therefore expand the random driving in eigenmodes of the sc states,

$$
\eta(\xi, \tau)=\sum_{n} \epsilon_{n}(\tau) \cos \left(k_{n} \xi\right) .
$$

Following the fluctuation-dissipation theorem, we assume the power spectrum of the form

$$
\left\langle\epsilon_{n}(\tau) \epsilon_{n^{\prime}}\left(\tau^{\prime}\right)\right\rangle=\frac{\alpha k_{B} T}{\omega_{n}} \delta_{n n^{\prime}} \delta\left(\tau-\tau^{\prime}\right),
$$

with the frequencies $\omega_{n}=\left[\left(1-j_{\text {ext }}^{2}\right)^{1 / 2}+k_{n}^{2}\right]^{1 / 2}$ and a proportionality factor $\alpha$. This corresponds to so-called pink noise, where high-frequency fluctuations are suppressed [58,59].

In our simulations, we create a single realization of random functions $\left\{\epsilon_{n}(t)\right\}$, which we interpolate from random values drawn from a Gaussian distribution with zero mean and variance $\alpha k_{B} T / \omega_{n}$ with a temporal step size $\Delta \tau$ set to 1 , and $\alpha k_{B} T=0.005$. The summation in Eq. (C1) is terminated at $n=10$, corresponding to a frequency cutoff $\omega_{10} \approx 9.5$ for our parameters. This function is then fed into the equation of motion (1) where it acts as a random scattering potential. Its effect is shown in Fig. 9, where we calculate the reflectance $|r(\omega)|^{2}$ from the propagation of a plasma wave.

Panel (a) shows the signal from a short junction with length $L=3.3$ without noise. The left plot shows the time evolution of the external field $E_{\text {ext }}$ (gray) and the reflected field $E_{r}$ (red). This is used to obtain the reflectance $|r(\omega)|^{2}$, with $r=$ $E_{r} /\left(E_{r}-2 E_{\text {ext }}\right)$. The reflectance features clear resonances at the eigenmodes of the junction with wave vectors $k_{n}=n \pi / L$ with $n=0,1,2$.

Panel (b) shows the same simulations with finite fluctuations. The reflectance still has the same features; the noise merely adds random fluctuations on top of the signal.

Finally, in panel (c) we present the simulations without noise in a long junction with $L=100$. The eigenmodes now overlap entirely, and the reflectance perfectly coincides with the theoretical expectation, which we obtain from the frequency-dependent dielectric constant [60]:

$$
\epsilon(\omega)=\epsilon_{0}\left(\sqrt{1-j_{\mathrm{ext}}^{2}}-\frac{1}{\omega^{2}}+i \frac{\nu_{c}}{\omega}\right) .
$$


[1] H. Hirori, A. Doi, F. Blanchard, and K. Tanaka, Appl. Phys. Lett. 98, 091106 (2011).

[2] T. Kampfrath, A. Sell, G. Klatt, A. Pashkin, S. Mährlein, T. Dekorsy, M. Wolf, M. Fiebig, A. Leitenstorfer, and R. Huber, Nat. Photon. 5, 31 (2011).

[3] T. Kampfrath, K. Tanaka, and K. A. Nelson, Nat. Photon. 7, 680 (2013).

[4] D. Nicoletti and A. Cavalleri, Adv. Opt. Photon. 8, 401 (2016).

[5] M. Först, C. Manzoni, S. Kaiser, Y. Tomioka, Y. Tokura, R. Merlin, and A. Cavalleri, Nat. Phys. 7, 854 (2011).

[6] A. Subedi, A. Cavalleri, and A. Georges, Phys. Rev. B 89, 220301(R) (2014).

[7] M. Knap, M. Babadi, G. Refael, I. Martin, and E. Demler, Phys. Rev. B 94, 214504 (2016).

[8] M. A. Sentef, A. F. Kemper, A. Georges, and C. Kollath, Phys. Rev. B 93, 144506 (2016).

[9] D. A. Kennes, E. Y. Wilner, D. R. Reichman, and A. J. Millis, Nat. Phys. 13, 479 (2017).

[10] M. Först, R. I. Tobey, H. Bromberger, S. B. Wilkins, V. Khanna, A. D. Caviglia, Y.-D. Chuang, W. S. Lee, W. F. Schlotter, J. J. Turner, M. P. Minitti, O. Krupin, Z. J. Xu, J. S. Wen, G. D. Gu, S. S. Dhesi, A. Cavalleri, and J. P. Hill, Phys. Rev. Lett. 112, 157002 (2014).

[11] M. Först, A. Frano, S. Kaiser, R. Mankowsky, C. R. Hunt, J. J. Turner, G. L. Dakovski, M. P. Minitti, J. Robinson, T. Loew, M. Le Tacon, B. Keimer, J. P. Hill, A. Cavalleri, and S. S. Dhesi, Phys. Rev. B 90, 184514 (2014).

[12] R. Mankowsky, B. Liu, S. Rajasekaran, H. Y. Liu, D. Mou, X. J. Zhou, R. Merlin, M. Först, and A. Cavalleri, Phys. Rev. Lett. 118, 116402 (2017).

[13] T. F. Nova, A. Cartella, A. Cantaluppi, M. Först, D. Bossini, R. V. Mikhaylovskiy, A. V. Kimel, R. Merlin, and A. Cavalleri, Nat. Phys. 13, 132 (2017).

[14] M. Rini, R. Tobey, N. Dean, J. Itatani, Y. Tomioka, Y. Tokura, R. W. Schoenlein, and A. Cavalleri, Nature (London) 449, 72 (2007).

[15] M. Liu, H. Y. Hwang, H. Tao, A. C. Strikwerda, K. Fan, G. R. Keiser, A. J. Sternbach, K. G. West, S. Kittiwatanakul, J. Lu, S. A. Wolf, F. G. Omenetto, X. Zhang, K. A. Nelson, and R. D. Averitt, Nature (London) 487, 345 (2012).

[16] A. D. Caviglia, R. Scherwitzl, P. Popovich, W. Hu, H. Bromberger, R. Singla, M. Mitrano, M. C. Hoffmann, S. Kaiser, P. Zubko, S. Gariglio, J.-M. Triscone, M. Först, and A. Cavalleri, Phys. Rev. Lett. 108, 136801 (2012).

[17] M. Först, A. D. Caviglia, R. Scherwitzl, R. Mankowsky, P. Zubko, V. Khanna, H. Bromberger, S. B. Wilkins, Y.-D. Chuang, W. S. Lee, W. F. Schlotter, J. J. Turner, G. L. Dakovski, M. P. Minitti, J. Robinson, S. R. Clark, D. Jaksch, J.-M. Triscone, J. P. Hill, S. S. Dhesiand, and A. Cavalleri, Nat. Mater. 14, 883 (2015).

[18] M. Först, K. R. Beyerlein, R. Mankowsky, W. Hu, G. Mattoni, S. Catalano, M. Gibert, O. Yefanov, J. N. Clark, A. Frano, J. M. Glownia, M. Chollet, H. Lemke, B. Moser, S. P. Collins, S. S. Dhesi, A. D. Caviglia, J.-M. Triscone, and A. Cavalleri, Phys. Rev. Lett. 118, 027401 (2017).

[19] D. Fausti, R. I. Tobey, N. Dean, S. Kaiser, A. Dienst, M. C. Hoffmann, S. Pyon, T. Takayama, H. Takagi, and A. Cavalleri, Science 331, 189 (2011).
[20] W. Hu, S. Kaiser, D. Nicoletti, C. R. Hunt, I. Gierz, M. C. Hoffmann, M. Le Tacon, T. Loew, B. Keimer, and A. Cavalleri, Nat. Mater. 13, 705 (2014).

[21] R. Mankowsky, A. Subedi, M. Först, S. O. Mariager, M. Chollet, H. T. Lemke, J. S. Robinson, J. M. Glownia, M. P. Minitti, A. Frano, M. Fechner, N. A. Spaldin, T. Loew, B. Keimer, A. Georgesand, and A. Cavalleri, Nature (London) 516, 71 (2014).

[22] M. Mitrano, A. Cantaluppi, D. Nicoletti, S. Kaiser, A. Perucchi, S. Lupi, P. Di Pietro, D. Pontiroli, M. Riccò, S. R. Clark, D. Jaksch, and A. Cavalleri, Nature (London) 530, 461 (2016).

[23] S. Savel'ev, A. L. Rakhmanov, and F. Nori, Phys. Rev. Lett. 94, 157004 (2005).

[24] S. Savel'ev, V. Yampolskii, and F. Nori, Phys. Rev. Lett. 95, 187002 (2005).

[25] S. Savel'ev, A. L. Rakhmanov, V. A. Yampol'skii, and F. Nori, Nat. Phys. 2, 521 (2006).

[26] L. N. Bulaevskii, A. E. Koshelev, and M. Tachiki, Phys. Rev. B 78, 224519 (2008)

[27] S. Z. Lin and X. Hu, Phys. Rev. B 82, 020504 (2010).

[28] S. Savel'ev, V. A. Yampol'skii, A. L. Rakhmanov, and F. Nori, Rep. Prog. Phys. 73, 026501 (2010).

[29] G. Guarcello, K. Fedorov, D. Valenti, B. Spagnolo, and A. Ustinov, arXiv:1501.04037v2.

[30] A. Dienst, M. C. Hoffmann, D. Fausti, J. C. Petersen, S. Pyon, T. Takayama, H. Takagi, and A. Cavalleri, Nat. Photon. 5, 485 (2011).

[31] A. Dienst, E. Casandruc, D. Fausti, L. Zhang, M. Eckstein, M. Hoffmann, V. Khanna, N. Dean, M. Gensch, S. Winnerl, W. Seidel, S. Pyon, T. Takayama, H. Takagi, and A. Cavalleri, Nat. Mater. 12, 535 (2013).

[32] S. Rajasekaran, E. Casandruc, Y. Laplace, D. Nicoletti, G. D. Gu, S. R. Clark, D. Jaksch, and A. Cavalleri, Nat. Phys. 12, 1012 (2016).

[33] R. Kleiner, F. Steinmeyer, G. Kunkel, and P. Müller, Phys. Rev. Lett. 68, 2394 (1992).

[34] L. Ozyuzer, A. E. Koshelev, C. Kurter, N. Gopalsami, Q. Li, M. Tachiki, K. Kadowaki, T. Yamamoto, H. Minami, H. Yamaguchi, T. Tachiki, K. E. Gray, W.-K. Kwok, and U. Welp, Science 318 1291 (2007).

[35] H. B. Wang, S. Guénon, B. Gross, J. Yuan, Z. G. Jiang, Y. Y. Zhong, M. Grünzweig, A. Iishi, P. H. Wu, T. Hatano, D. Koelle, and R. Kleiner, Phys. Rev. Lett. 105, 057002 (2010).

[36] L. N. Bulaevskii and A. E. Koshelev, Phys. Rev. Lett. 99, 057002 (2007).

[37] S. Lin and X. Hu, Phys. Rev. Lett. 100, 247006 (2008).

[38] S. Lin and X. Hu, Phys. Rev. B 79, 104507 (2009).

[39] M. Tachiki, S. Fukuya, and T. Koyama, Phys. Rev. Lett. 102, 127002 (2009).

[40] A. E. Koshelev, Phys. Rev. B 78, 174509 (2008).

[41] T. Koyama, H. Matsumoto, M. Machida, and K. Kadowaki, Phys. Rev. B 79, 104522 (2009).

[42] Y. Nonomura, Phys. Rev. B 80, 140506(R) (2009).

[43] X. Hu and S.-Z. Lin, Supercond. Sci. Technol. 23, 053001 (2010).

[44] U. Welp, K. Kadowaki, and R. Kleiner, Nat. Photon. 7, 702 (2013). 
[45] R. Ansorge and M. Graves, The Physics and Mathematics of MRI (Morgan \& Claypool Publishers, San Rafael, USA, 2016).

[46] A. E. Koshelev and I. S. Aranson, Phys. Rev. Lett. 85, 3938 (2000).

[47] A. E. Koshelev and I. S. Aranson, Phys. Rev. B 64, 174508 (2001).

[48] L. N. Bulaevski and A. E. Koshelev, J. Supercond. Novel Magn. 19, 349 (2006).

[49] Lawrence Livermore National Lab, IDA package.

[50] M. Salerno, E. Joergensen, and M. R. Samuelsen, Phys. Rev. B 30, 2635 (1984).

[51] According to the AC Josephson relation, $d \phi / d \tau$ gives the local voltage in the junction.

[52] D. W. McLaughlin and A. C. Scott, Phys. Rev. A 18, 1652 (1978).

[53] F. Geniet and J. Leon, Phys. Rev. Lett. 89, 134102 (2002).
[54] S. J. Denny, S. R. Clark, Y. Laplace, A. Cavalleri, and D. Jaksch, Phys. Rev. Lett. 114, 137001 (2015).

[55] R. Höppner, B. Zhu, T. Rexin, A. Cavalleri, and L. Mathey, Phys. Rev. B 91, 104507 (2015).

[56] J.-I. Okamoto, A. Cavalleri, and L. Mathey, Phys. Rev. Lett. 117, 227001 (2016).

[57] M. B. Fogel, S. E. Trullinger, A. R. Bishop, and J. A. Krumhansl, Phys. Rev. B 15, 1578 (1977).

[58] M. B. Weissman, Rev. Mod. Phys. 60, 537 (1988).

[59] We also checked numerically the influence of white noise, i.e., $\left\langle\epsilon_{n}(\tau) \epsilon_{n^{\prime}}\left(\tau^{\prime}\right)\right\rangle=\alpha k_{B} T \delta_{n n^{\prime}} \delta\left(\tau-\tau^{\prime}\right)$, with a sharp cutoff at $\omega=10$. It did not affect the results of the time evolutions in this publication. Generally, we note that the noise can slightly move the boundaries in Figs. 5-7, i.e., it can influence the switching near criticality, but it does not affect the larger structure of the plots.

[60] A. E. Koshelev, Phys. Rev. B 76, 054525 (2007). 